\title{
Anabases
}

ANABASES Traditions et réceptions de l'Antiquité

$15 \mid 2012$

Varia

\section{Pierre Vidal-Naquet, historien engagé. Autour de L'affaire Audin}

Pauline Schmitt Pantel

\section{(2) OpenEdition}

1 Journals

Édition électronique

URL : http://journals.openedition.org/anabases/3631

DOI : 10.4000/anabases.3631

ISSN : 2256-9421

Éditeur

E.R.A.S.M.E.

Édition imprimée

Date de publication : 1 avril 2012

Pagination : 11-25

ISSN : 1774-4296

\section{Référence électronique}

Pauline Schmitt Pantel, «Pierre Vidal-Naquet, historien engagé. Autour de L'affaire Audin », Anabases

[En ligne], 15 | 2012, mis en ligne le 01 avril 2015, consulté le 21 octobre 2019. URL : http:// journals.openedition.org/anabases/3631 ; DOI : 10.4000/anabases.3631

(c) Anabases 
Anabases 15 (2012), p. 11-25

\section{Pierre Vidal-Naquet, historien engagé. Autour de L'affaire Audin}

Pauline Schmitt Pantel

Le 18 MARs 1958, il y a jour pour jour cinquante-trois ans, est organisé à Paris un " meeting de masse " du comité Audin (avec le centre du Landy et la ligue des droits de l'homme) ${ }^{1}$. Dans sa lettre du $1^{\text {er }}$ mars 1958, Pierre Vidal-Naquet avait annoncé ce meeting à Josette Audin, la veuve du jeune mathématicien disparu depuis le 21 juin 1957 à Alger. Je cite :

"Le comité Audin serait heureux de recevoir un message (de votre part) pour les meetings de masse qu'il organise (avec) le centre du Landy et la ligue des droits de l'homme (à Paris le 18 mars, à Caen le 18 mars, à Marseille le 19 mars). Le mieux évidemment aurait été que vous puissiez venir vous-même (le comité Audin vous aurait déchargée de tous frais). Mais peut-être est-ce impossible ? Le meeting de Paris sera présidé par Laurent Schwartz et les orateurs seront Daniel Mayer, Marcel Paul, P.H. Simon, Michel Bruguier, Jean-Paul Sartre, Madame Alleg. Il va de soi que toute cette action ne prend véritablement son sens que dans le cadre général de la lutte contre la guerre d'Algérie. Certains signes font croire que cette lutte peut enfin reprendre avec ampleur, notamment

1 La journée d'étude « Les Antiquisants français au xx siècle : parcours d'historiens engagés " a eu lieu le 18 mars 2011 à l'université de Toulouse-Le Mirail. Le comité Maurice Audin a été créé en novembre 1957 à Paris à l'initiative de plusieurs jeunes enseignants dont Jean Gillet assistant à la Sorbonne, Michel Crouzet, Luc Montagnier... Jacques Fernand Cahen (La Flèche) avança l'idée du Comité. Il se dota peu à peu d'instances dont un secrétariat : Crouzet, Montagnier, Panigel, Pierre Vidal-Naquet, d'un président : Albert Châtelet puis Laurent Schwartz, de vice-présidents : Dresch, Marrou... Le centre du Landy ou " Comité de défense des Libertés et de la Paix " publiait la revue Témoignages et documents. 
dans les milieux ouvriers où elle était considérablement ralentie. Nous comptons donc sur vous et en attendant je vous dis mon amitié ${ }^{2}$."

Ces meetings ont lieu à un moment important : Pierre Vidal-Naquet est en train de terminer " la petite brochure ", comme il l'appelle, qu'il écrit sur l'affaire Audin. C'est de l'écriture de ce premier livre de Pierre Vidal-Naquet historien que je voudrais parler aujourd'hui. Pourquoi ce choix?

Il y a à cela plusieurs raisons.

L'affaire Audin est le premier livre d'histoire écrit par Pierre Vidal-Naquet. La couverture de l'édition de 1958 porte : Pierre Vidal-Naquet, agrégé d'histoire. Né en juillet 1930, il a alors un peu moins de vingt-huit ans. Il a passé l'agrégation d'histoire en juin 1955, a été professeur de lycée à Orléans en 1955-1956, puis assistant à l'université de Caen en octobre 1956. L'affaire Audin est aussi le livre qui marque le début d'un engagement citoyen qui va durer toute sa vie.

Dès ces années-là Pierre Vidal-Naquet a un sujet de thèse en histoire grecque qui lui a été donné par le professeur d'histoire grecque à la Sorbonne, André Aymard (sur l'historiographie grecque au IV siècle avant J.-C.), et il enseigne l'histoire grecque à l'université de Caen, mais il n'a encore rien publié dans le domaine de l'histoire ancienne. En 1956-1958 il écrit deux articles qui vont paraitre dans des revues en 1960 et seront repris dans son livre Le Chasseur noir en $1981^{3}$.

L'affaire Audin a été sans doute reçue différemment selon les époques et selon les lecteurs. Pour ma génération, à l'université de Lyon où Pierre Vidal-Naquet était professeur d'histoire grecque entre 1964 et 1966, ce livre, ainsi que l'engagement contre la guerre d'Algérie et contre la torture, conféraient à son auteur un statut, voire une " aura " d'historien différent des autres enseignants, beaucoup plus bien sûr que son premier livre d'histoire grecque Clisthène l'Athénien, écrit avec Pierre Lévêque, qui venait juste de paraître (en 1964) ${ }^{4}$. Dans les couloirs de la vieille faculté de lettres du quai du Rhône, dans les réunions de l'association des étudiants d'histoire, une sérieuse réputation précédait Pierre Vidal-Naquet qui avait alors entre trente-quatre et trentesix ans, et ce n'était pas encore celle de l'helléniste spécialiste des marges, mais bien celle d'un intellectuel qui savait dire "non ». Pour de jeunes étudiants c'était important. Pour ses futurs élèves bien sûr aussi.

2 Daniel Meyer : Ligue des droits de l'homme. Marcel Paul : membre de la CGT et du PC. Michel Bruguier : avocat, ancien dirigeant FFI du Gard.

3 P. VIDAL-NAQUET, "Essai sur quelques aspects de l'expérience temporelle des Grecs ", Revue de l'histoire des religions, 1960, p. 55-80 et "Epaminondas pythagoricien ou le problème tactique de la droite et de la gauche ", en collaboration avec P. LÉvêQUE, Historia 9 (1960), p. 294-308.

4 Il faut citer le titre en entier : Clisthène l'Athénien. Essai sur la représentation de l'espace et du temps dans la pensée politique grecque de la fin du vI siècle à la mort de Platon. Besançon et Paris, Les Belles Lettres, 1964. 
Faire un rapprochement et tenter une comparaison entre la méthode et le contenu des premiers articles de Pierre Vidal-Naquet sur la Grèce et L'affaire Audin n'a pas beaucoup de sens. En revanche si l'on considère l'ensemble de son œuvre, sur près de cinquante ans, le rapport entre les interrogations sur le présent et la manière de voir le passé et éventuellement l'inverse peuvent être étudiés 5 . De nombreux entretiens, articles et livres sont parus sur ce thème, ainsi que plusieurs émissions de radio et de télévision. On peut en faire la synthèse mais il est difficile de dire des choses nouvelles. Ceci en grande partie aussi parce que Pierre Vidal-Naquet a lui-même réfléchi à la question que nous posons aujourd'hui et nous offre donc un fil de lecture. Comme le rappelle François Hartog, «nul historien contemporain n'a plus écrit sur lui-même, soucieux qu'il était de présenter, d'expliquer, de fixer l'histoire de sa vie, anxieux de recueillir les traces de ses engagements d'historien ${ }^{6}$ ".

Je vais suivre ce fil de lecture dans les Mémoires ${ }^{7}$ et dans un livre au titre explicite, Le Choix de l'histoire ${ }^{8}$. Puis je reviendrai à L'affaire Audin. Le livre Le Choix de l'histoire, consiste en la republication de quatre textes dont deux concernent plus particulièrement notre thème : "Esquisse d'un parcours anticolonialiste" " et "Pourquoi et comment je suis devenu historien ${ }^{10}$ ». Sous des titres sensiblement différents, l'un insistant sur l'engagement citoyen, l'autre sur la profession d'historien, les deux textes abordent en fait les mêmes thèmes. Et tous les deux présentent essentiellement les années de formation. Ainsi ils sont complémentaires et dans une large mesure également conformes à ce qu'écrit Pierre Vidal-Naquet dans ses Mémoires. Il est frappant de voir à quel point les deux lignes de force de sa vie, l'engagement citoyen et l'écriture de l'histoire, sont tissées l'une avec l'autre.

Le texte le plus ancien : «Esquisse d'un parcours anticolonialiste » me sert de pivot. Le passé personnel, l'histoire de vie, de Pierre Vidal-Naquet le prépare à l'anticolonialisme. Il grandit dans une famille juive assimilée, de tradition patriotique, républicaine et dreyfusarde. Le récit de l'affaire Dreyfus que lui fit son père en 1942 ou 1943 joue un rôle important dans sa formation. De l'affaire Dreyfus, Pierre Vidal-Naquet conserve l'idée de la victoire sur l'erreur et l'injustice. Le texte de Chateaubriand qu'il connaît très tôt est sa description modèle de la fonction de l'historien :

"Lorsque dans le silence de l'abjection on n'entend plus que la chaîne de l'esclave et la voix du délateur ; lorsque tout tremble devant le tyran, et qu'il est aussi dangereux

5 P. Schmitt Pantel, "Pierre Vidal-Naquet", in Les Historiens, Paris, Armand Colin, 2003, p. 317-335.

6 F. HarTog, Vidal-Naquet, historien en personne, Paris, La Découverte, 2007, p. 7.

7 Mémoires 1, Paris, Seuil/La Découverte, 1995 ; Mémoires 2, Paris, Seuil/La Découverte, 1998.

8 P. Vidal-Naquet, Le Choix de l'histoire, Paris, Arléa, 2004.

9 Texte paru dans la Revue d'études palestiniennes, juin 2001.

10 Texte paru dans Esprit, août-septembre 2003. 
d'encourir sa faveur que de mériter sa disgrâce, l'historien parait, chargé de la vengeance des peuples ${ }^{11}$."

Scout chez les Éclaireurs unionistes, il reconnaît l'influence du protestantisme qui lui donne l'" occasion de se familiariser avec la Bible que les catholiques pratiquaient alors très peu. La Bible est à sa façon un livre d'histoire que les protestants lisent en pratiquant le libre examen ${ }^{12}$ ". Dès 1946-1947, Pierre Vidal-Naquet alors lycéen devient partisan de la renonciation au pouvoir colonial, il se définit comme un homme de gauche, ni socialiste ni communiste. Dans ces années-là s'affirme son goût pour l'histoire contemporaine ${ }^{13}$. Il a alors l'idée de faire un travail sur la guerre d'Espagne. Puis il découvre la Grèce (le pays) et Platon en rédigeant un mémoire sur Platon et l'histoire avec Henri-Irénée Marrou en 1953. Il travaille en même temps sur la première élection de Jaurès à Carmaux (le 8 janvier 1893) avec Ernest Labrousse.

"Ainsi s'étaient établis les deux côtés de mon travail à cette époque ancienne : le côté de chez Jaurès et le côté de chez Platon. D'autres viendront s'y ajouter, mais en choisissant l'histoire grecque j'avais l'espoir ou l'illusion que j'échapperais à la tyrannie de l'immédiat. Du moins ma passion pour la Grèce lointaine et pour Platon en particulier me donnaitelle une sorte de distance que ne m'aurait assurément pas donnée un travail sur la guerre d'Espagne ${ }^{14}$."

En 1953-1954 le problème de la torture en Indochine et en Algérie est soulevé par François Mauriac et Claude Bourdet. L'horreur de la torture a chez Pierre VidalNaquet des résonances personnelles : son père a été torturé par la Gestapo en mai 1944. Le témoignage de son ami de lycée Robert Bonnaud, soldat appelé en Algérie, sur ce qu'il a vu dans les Némentchas (en 1956), paru dans Esprit en avril 1957, joue le rôle de déclencheur. La publication de ce témoignage ne provoque en effet aucun scandale en France. Pierre Vidal-Naquet en est abasourdi. "Ma machine personnelle était lancée ", écrit-il ${ }^{15}$. Dans ce contexte surgit l'annonce de la mort sous la torture de Maurice Audin en juin 1957. Pierre Vidal-Naquet propose à Josette Audin, dès septembre 1957, "d'écrire moi-même une brochure montrant les difficultés que soulevait la version officielle de l'évasion ${ }^{16}{ }$. C'est L'affaire Audin publiée en mai 1958.

À cette époque Pierre Vidal-Naquet se voit comme un mélange des trois sortes d'opposants à la guerre d'Algérie : les héritiers de l'Affaire Dreyfus, les bolcheviks et les tiers-mondistes. Sa carrière d'historien de l'antiquité subit des à-coups : il est nommé à Caen puis suspendu après la signature du manifeste des 121 qui reconnaissait le droit

11 Le Choix de l'histoire, p. 16-17 et p. 56.

12 Ibid., p. 18.

13 Ibid., p. 19.

14 Ibid., p. 27.

15 Ibid., p. 34 et p. 62. Citation p. 62.

16 Ibid., p. 35 et p. 63. Citation p. 63. 
à l'insoumission dans la guerre d'Algérie, en 1960-1961. Il est ensuite nommé à Lille. Dans ces années-là, Pierre Vidal-Naquet se définit comme un activiste qui, au sein du comité Audin, milite contre la torture, et rédige des articles pour Témoignages et documents puis pour Vérité Liberté, et comme un historien dont le champ d'étude est une guerre contemporaine. Il s'agit pour lui de démontrer que l'appareil d'État dans sa quasi-totalité est impliqué dans la pratique de la torture. Il prépare et rédige La Raison d'État, recueil de documents officiels, paru en avril 1962. Un court récit tiré de ce livre est publié en anglais. Il devient La Torture dans la République en 1972.

Ainsi les années de formation forgent le lien qui ne se démentira pas tout au long de la vie de Pierre Vidal-Naquet entre l'étude du passé et l'étude liée à l'engagement citoyen dans le présent. Pierre Vidal-Naquet a-t-il tenté d'expliquer ce lien ?

Le texte de présentation de notre journée d'études rappelle cette phrase tirée de ses Mémoires que je cite en entier :

"Y a-t-il un rapport entre mon activité d'historien de la Grèce et mon activité publique, voire politique ? La réponse est un oui, nuancé comme il se doit. Assurément je me suis arc-bouté sur la Grèce, dès 1951, pour éviter de tomber dans le piège de l'immédiateté. Cela dit, ce n'est pas tout à fait par hasard si, aidé de Pierre Lévêque, j'ai choisi comme sujet de mon premier livre "grec" le personnage de Clisthène, fondateur à la fin du VI ${ }^{\mathrm{e}}$ siècle avant J.-C. de ce qu'on appellera au siècle suivant la démocratie. Je suis autant qu'un autre conscient de la différence énorme qui sépare la démocratie athénienne, directe, écartant femmes, étrangers, esclaves, de la nôtre qui est représentative. Il n'empêche, les mots ont leur importance, et celui de démocratie a eu une sacrée postérité. Loin de quitter le contemporain, j'acquérais sur lui un regard sans doute distancié, mais, je l'espère, lucide ${ }^{17}$."

Pierre Vidal-Naquet se demande ailleurs :

"Y a-t-il une différence de nature entre mes écrits d'histoire contemporaine et mes écrits d'histoire ancienne ? Il est vrai que les premiers sont plus caractérisés par l'opposition mensonge/vérité que les seconds... Je ne crois pas à la vérité absolue mais je crois beaucoup à la nécessité de débusquer l'imposture même à propos de l'Atlantide ${ }^{18}$. "

Le premier exemple que donne Pierre Vidal-Naquet de ce double intérêt d'historien pour le passé et pour le contemporain est général, et de nombreux historiens pourraient y souscrire. Son second exemple est plus précis, plus personnel aussi. Il s'agit de la tragédie, mais pas seulement de la tragédie grecque, de celle de Shakespeare aussi.

" J'ai utilisé le modèle tragique et plus spécifiquement shakespearien pour comprendre les procès de l'Est, spectacles offerts au public comme les combats de gladiateurs à Rome

17 P. Vidal-Naquet, Mémoires 2, p. 239.

18 P. Vidal-Naquet, Le Choix de l'histoire, p. 49. 
et se terminant par la mort du héros dégradé... Tragédie ou histoire, il m’est arrivé de poser cette question. Tragédie et histoire, il m'est arrivé de vivre cette association et de réfléchir sur elle ${ }^{19}$. "

On conviendra qu'il ne s'agit pas là non plus d'une démarche comparatiste bien ciblée et circonscrite, mais d'une histoire de vie, la tragédie ayant frappé Pierre VidalNaquet, ses frères et sa sœur le 15 mai 1944 avec l'arrestation à Marseille de leurs parents, leur déportation à Auschwitz et leur mort.

Bref, Pierre Vidal-Naquet aborde la question du rapport entre son métier d'historien de la Grèce et son activité publique sans vraiment y répondre. Il y revient toutefois à l'extrême fin de ses Mémoires parce que c'était certainement une question qu'on lui posait sans cesse, dans chaque interview et chaque entretien et qu'il fallait bien qu'il s'en débarrasse. Mais d'une certaine manière il va la contourner une nouvelle fois en s'interrogeant moins sur le rapport entre engagement politique et recherche sur la Grèce antique que sur sa propre méthode d'historien.

«Un des paradoxes de ma vie d'historien est qu'elle s'est déroulée sur deux plans. Dans mon domaine propre, celui de l'histoire grecque, c'est à l'étude des représentations que je me suis consacré. C'est dire là que, dans ce registre, je me préoccupais assez peu de l'établissement des faits. Un système de pensée n'est pas exactement un fait au sens classique du $\operatorname{mot}^{20}$. Or, dans un autre domaine, celui de l'histoire contemporaine, je n'ai pas cessé au contraire depuis L'affaire Audin (1958) jusqu'aux Assassins de la mémoire (1987) de chercher à établir des vérités au sens le plus élémentaire de ce mot : Audin s'est-il évadé ou a-t-il été tué ? Les chambres à gaz hitlériennes ont-elles été une fiction ou une sinistre réalité ? Entre ces deux domaines je n'ai cessé d'établir des passerelles. Un lecteur attentif trouvera des citations de Thucydide dans La Torture dans la République (1972) comme dans Les Assassins de la mémoire. Cela dit, cette contradiction, je l'assume et j'en tire même quelque fierté ${ }^{21}$."

Dans l'analyse de son métier d'historien du présent et du passé Pierre VidalNaquet souligne deux traits constants : il a cherché quand cela est possible à établir des passerelles entre l'histoire ancienne et l'histoire contemporaine ; l'étude sur Jean Moulin est un bon exemple ${ }^{22}$, celle sur Flavius Joèphe également ${ }^{23}$. Et il a mis en place des processus d'écriture de l'histoire relativement différents en rapport avec les sujets

19 P. Vidal-Naquet, Mémoires 2, p. 240.

20 Pierre Vidal-Naquet dira ailleurs avec ironie qu'il n'est guère intéressant de situer l'Acropole. Voir le numéro 157-158 (2006) de Raison Présente, Le " fait " en histoire, dédié à Pierre Vidal-Naquet.

21 P. Vidal-Naquet, Mémoires 2, p. 369-370.

22 Le trait empoisonné. Réflexions sur l'affaire Jean Moulin, Paris, La Découverte, 1993.

23 Préface à La guerre des Juifs. Voir P. Schmitt Pantel, "Pierre Vidal-Naquet ", in Les Historiens, p. 317-335. 
traités, qui ne sont pas non plus comparables. Clisthène l'Athénien n'est pas L'affaire Audin.

Revenons à l'Affaire Audin qui est un exemple modèle du double engagement militant et historien ${ }^{24}$. Pour débusquer dans le premier livre écrit par Pierre VidalNaquet la méthode de l'historien, nous disposons de nombreuses sources. L'affaire Audin paraît le 12 mai 1958 avec une préface de Laurent Schwartz qui reprend son article paru dans l'Express du 16 janvier 1958. Le 13 mai 1958 le général Massu prend la présidence d'un comité de salut public à Alger. L'affaire Audin paraît avec une bande annonce portant : "Massu et ses hommes ", bande qui avait été d'abord rédigée sous la forme "Massu et ses complices ». Treize mille exemplaires sont diffusés en quelques semaines. Ce petit livre est republié dans un livre beaucoup plus épais intitulé également L'Affaire Audin en novembre 1989, qui comprend, outre le texte initial, deux autres parties : la première, "Disparition ", expose comment naquit et se développa l'affaire Audin, la troisième, "Chronique d'un déni de justice ", expose l'évolution de l'affaire et ses prolongements judiciaires, politiques et moraux. Pierre Vidal-Naquet a parlé de ce livre à de nombreuses reprises et en particulier dans ses Mémoires.

Une autre source est très intéressante : la correspondance entre Josette Audin et Pierre Vidal-Naquet du 9 septembre 1957 à la parution du livre en mai $1958^{25}$. J'ai, dans un premier temps, pu consulter les lettres écrites par Pierre Vidal-Naquet, lettres qui avaient été conservées par Josette Audin : cette dernière en avait donné une copie à Geneviève Vidal-Naquet qui m'a permis de les lire. En septembre 2011 j’ai eu accès aussi à la lecture d'un petit nombre de lettres de Josette Audin, conservées dans les archives de Pierre Vidal-Naquet ${ }^{26}$. Lire cette correspondance croisée est très émouvant ${ }^{27}$. Cette correspondance éclaire le travail commun avec Josette Audin qui a permis la rédaction de L'affaire Audin ${ }^{28}$. Elle permet de repérer aussi le moment où Pierre Vidal-Naquet a pris l'habit d'historien.

Cette brochure est son premier livre d'historien. La manière dont il l'a conçue, fabriquée, écrite, apparaît clairement dans cette correspondance qui nous permet de toucher du doigt les étapes et les principes de la fabrique de l'histoire. Bertrand

24 Voir l'analyse de P. Pachet, "D’un style politique ", in F. Hartog, P. Schmitt, A. Schnapp (dir.), Pierre Vidal-Naquet, un historien dans la cité, Paris, La Découverte, 1998, p. 67-83.

25 Cette correspondance dure en fait presque cinquante ans puisque la dernière lettre de Pierre Vidal-Naquet à Josette Audin date du 11 janvier 2006.

26 Fonds d'archives Pierre Vidal-Naquet, École des hautes études en sciences sociales. Sur la constitution de ce fonds, voir Goulven LE BRECH, « Les archives des chercheurs en sciences humaines et sociales : l'exemple du fonds Pierre Vidal-Naquet (Service des archives de l'EHESS) ", La Gazette des Archives, no 212-4 (2008), p. 69-77.

27 Je remercie Josette Audin et Geneviève Vidal-Naquet de m'avoir permis cette lecture.

28 J'ai pour ma part consulté l'ensemble du dossier d'archives concernant l'affaire Audin dans le Fonds d'archives Pierre Vidal-Naquet. 
Hamelin, qui avait lu les lettres de Pierre Vidal-Naquet, en a indiqué les points forts que j'avais de mon côté également repérés ${ }^{29}$. En résumé, il relève la qualité d'agrégé d'histoire dont Pierre Vidal-Naquet se prévaut, le propos de Jean-Paul Sartre qui souligne "des méthodes d'historien ", et aussi l'aspect transgressif, selon lui, qui consiste pour un historien de l'antiquité à s'intéresser au temps présent. La première tâche de l'historien est la collecte de sources : il faut regrouper les pièces concernant l'affaire, les documents officiels, les textes publiés, la Question d'Henri Alleg qui a été publiée en février 1958 aux éditions de Minuit est en revanche peu citée. Toutefois la brochure ne sera pas une collection annotée de documents. Bertrand Hamelin s'interroge sur le statut de ce texte qui ne laisse pas passer l'émotion et refuse le ton du pamphlétaire ou du militant, un texte où toute allusion politique directe est absente. Il souligne enfin qu'il est difficile de mesurer la part de Jérôme Lindon dans la rédaction finale et rappelle l'importance d'une publication de L'affaire Audin aux Éditions de Minuit, maison d'édition militante.

Je voudrais pour ma part revenir sur un point que révèle cette correspondance : l'usage fait par Pierre Vidal-Naquet du récit que donne Josette Audin de l'arrestation de son mari et des jours qui ont suivi à Alger. Après lui avoir assigné une place indépendante et importante au début de la brochure dans un premier état de la rédaction, Pierre Vidal-Naquet fait disparaître ce témoignage en tant que tel dans la version publiée. Si l'on suit le long cheminement qui a abouti à cette disparition, on peut trouver, je crois, en germe tout ce qui est au cœur de la méthode historique de Pierre Vidal-Naquet.

Comment le projet a-t-il évolué ?

Dans la première lettre de Pierre Vidal-Naquet à Josette Audin le 9 septembre 1957, il est question de constituer un dossier avec le témoignage de Josette Audin et les pièces essentielles de l'affaire. Le contenu de ce dossier est précisé dans la lettre du 9 octobre : un récit de Josette Audin, les pièces diverses en sa possession, les protestations syndicales... Le 28 octobre, Josette Audin accuse réception des lettres précédentes et dit son intention de rassembler les pièces utiles pour la constitution du dossier.

Dans sa lettre du 30 octobre Pierre Vidal-Naquet se dit disposé à s'occuper de la brochure concernant Maurice Audin, et le 24 novembre la nature de la brochure est précisée : "Quelque chose de consistant, un véritable petit livre qui puisse frapper les esprits $^{30}$. " Le 26 novembre, Josette Audin remercie Pierre Vidal-Naquet d'accepter de s'occuper de la publication de la brochure. "Je vous fais bien sûr confiance pour vous en charger ». Elle annonce sa venue à Paris : "Je serai à votre disposition pour vous

29 B. Hamelin, "Écrire en historien : Pierre Vidal-Naquet, L'affaire Audin», in Th. Augais, M. Hisum ет Сн. Michel, Écrire et publier la guerre d'Algérie : de l'urgence aux résurgences, Paris, Éditions Kimé, 2010, p. 69-82. Je n'ai pris connaissance de cet article qu'une fois ma propre recherche achevée.

30 Lettre P. V.N. du 24 novembre 1957. Les lettres de Josette Audin sont abrégées en : J.A. Les lettres de Pierre Vidal-Naquet sont abrégées en : P. V.N. 
donner de vive voix d'autres précisions et d'une façon générale tous les renseignements dont vous auriez besoin ${ }^{31}$."

Dans la lettre suivante du 26 décembre 1957, Pierre Vidal-Naquet annonce qu'il est chargé par le comité Audin de rédiger la brochure sous le contrôle d'une commission. Il ajoute :

"Nous avons pensé qu'il fallait faire les choses le plus objectivement possible : aussi pensons-nous que vous devez vous-même présenter les faits dont vous avez été vousmême le témoin. Acceptez-vous de rédiger une fois de plus un récit minutieux de ces événements : arrestation, visite des parachutistes du 22 juin, entrevue avec le colonel Trinquier ? Un récit paraissant sous votre signature aurait plus de poids que tout autre. Immédiatement après viendrait la présentation et l'analyse critique des documents qui sont en notre possession, c'est ce travail dont je me chargerai ${ }^{32}$."

Le 2 janvier 1958 Josette Audin répond : "Je suis évidemment d'accord pour ce que vous me dites au sujet de la brochure, mais j’ai déjà plusieurs fois rédigé le récit des événements. Est-ce cela que vous désirez ou avez-vous besoin d'une autre rédaction $^{33}$ ? » Pierre Vidal-Naquet précise alors (lettre du 8 janvier) :

" Je sais que vous avez rédigé plusieurs fois le récit des événements et j’ai un de ces récits. Ce n'est pas tout à fait cela dont j'ai besoin bien que vous puissiez garder des paragraphes entiers de ce récit. Il s'agit d'énumérer jour par jour et avec toutes les précisions imaginables les faits dont vous avez été le seul témoin : arrestation, surveillance, visite des parachutistes, entrevues avec la commission de sauvegarde, avec le commissaire de police, avec le colonel Trinquier etc. Ce récit peut être aussi long que vous voulez, plus long en tout cas que celui que j'ai. Le modèle serait le récit oral que vous m’avez fait. Je vous serais très reconnaissant d'accepter ce petit travail qui est capital ${ }^{34}$. "

Josette Audin dans la lettre du 11 janvier : "Je vous envoie un nouveau récit, je pense que cela ira. Sinon dites-moi ce qui ne va pas, je le modifierai ${ }^{35}$. "Pierre Vidal-Naquet répond : "Votre récit est tout à fait ce qu'il faut avec quelques réserves. Permettez-moi d'en éliminer ce qui n'est pas témoignage mais appel à l'opinion publique $^{36}$. » Dans la lettre du 21 janvier, Pierre Vidal-Naquet annonce que le plan de la brochure est arrêté :

31 Lettre J.A. du 26 novembre 1957. Josette Audin est présente à Paris lors de la première conférence de presse du Comité Audin, présidée par Louis Gernet, et à la soutenance in absentia de la thèse de Maurice Audin le 2 décembre 1957 à la Sorbonne.

32 Lettre P. V.N., 26 décembre 1957.

33 Lettre J.A, 2 janvier 1958.

34 Lettre P. V.N., 8 janvier 1958. Les mots soulignés le sont dans la lettre de P. V.N.

35 Lettre J.A., 11 janvier 1958.

36 Lettre P. V.N., 14 janvier 1958. 
«1. Biographie

2. Votre récit

3. Thèse officielle ou thèses officielles ?

4. La réalité des tortures. »

Aucune lettre de Josette Audin n'est conservée entre le 21 janvier et le 12 avril $1958^{37}$. Durant ce laps de temps, les quelques lettres conservées de Pierre VidalNaquet ne portent pas sur la rédaction de la brochure mais sur l'invitation de Josette Audin par le comité Audin à venir à Paris en personne ou à envoyer un message pour le prochain meeting organisé par le comité. En avril l'échange de lettres reprend autour de la rédaction de la brochure. Le 9 avril Pierre Vidal-Naquet écrit : "Mon travail n'a plus besoin que d'une dernière révision. Je répète qu'il apporte des certitudes. N'oubliez pas que la méthode historique existe. Voudriez-vous enfin avoir l'extrême gentillesse de me renvoyer votre récit qui a été stupidement égaré ${ }^{38}$. " Le 12 avril Josette Audin renvoie le récit. Le lendemain, 13 avril Pierre Vidal-Naquet écrit : "Je vous signale que mon travail est maintenant achevé. Vous aurez mon travail très prochainement. Il ne fait appel à aucun élément qui ne figure au dossier. C'est de l'interprétation de celui-ci par la méthode critique que naît la lumière. Je répète que celle-ci est aveuglante ${ }^{39}$. "

Et voici un extrait de la lettre de Pierre Vidal-Naquet du 19 avril :

"Vous avez maintenant j'imagine mon texte. Ne le montrez, n'en parlez à personne. Ne vous étonnez pas de son caractère froid, "glacé" même, m’a dit Maître Borker. C'est une condition nécessaire à un travail historique qui doit être d'une objectivité absolue. Mes quelques erreurs que vous aurez pu relever auront déjà été corrigées à l'heure où vous lirez ces lignes. Vos avocats ont donné leur accord. Dans ces conditions tout ira très vite. Je vous remercie de vos deux lettres et des photos. C'est sans doute la photo d'identité qui sera reproduite. Merci d'avoir pris avec moi un certain nombre de responsabilités. Cette affaire n'existerait pas sans vous. Nous sommes fiers d'avoir travaillé avec vous ${ }^{40}$. "

Le 22 avril Josette Audin a reçu le texte et l'a lu :

"Je vous écris après notre conversation téléphonique où j'étais un peu gênée de vous parler du texte que vous avez écrit. Comme je vous l'ai dit, je suis bien sûr d'accord sur l'ensemble, une fois apportées cependant les corrections qu'a faites Maître Borker au début... J'ai été surprise de lire cette conclusion aussi. Mon point de vue est qu'il sera difficile d'avoir officiellement des renseignements sûrs tant que durera la guerre d'Algérie. C'est pourquoi j'aurais aimé voir ce texte se terminer en appelant à ce que l'on mette

37 Du moins dans le dossier conservé dans le Fonds d'archives Vidal-Naquet.

38 Lettre P. V.N., 9 avril 1958.

39 Lettre P. V.N., 13 avril 1958. Il est peu probable que le 13 avril Pierre Vidal-Naquet ait déjà reçu la nouvelle version du récit de Josette Audin qu'elle a envoyée d'Alger la veille.

40 Lettre P. V.N., 20 avril 1958. 
fin à la guerre d'Algérie, et avec elle, à tous ses produits : les lieutenants Charbonnier et autres tortionnaires. Il me reste à vous remercier du travail que vous avez fait pour regrouper et analyser les documents que l'on a obtenus depuis le mois de juin et prouver ainsi le mensonge des parachutistes et du gouvernement. J'espère que votre travail sera très utile ${ }^{41}$."

Dans la réponse de Pierre Vidal-Naquet du 28 avril 1958 on peut lire :

"Maître Borker et le comité sont maintenant d'accord pour ne pas insérer à la fin du livre l'appel dont vous parlez. Quel que soit votre accord à ce sujet il faut vous rappeler que le livre ne tire sa force que de son objectivité scientifique. Ce que nous ne faisons pas, la presse le fera. J'espère malgré tout que vous ne serez pas déçue ${ }^{42}$. "

La chronologie des lettres permet de préciser que la volonté de publier le témoignage de Josette Audin en second point de la brochure sous le titre de « récit " a disparu entre le 21 janvier 1958 et le 13 avril 1958.

Comment l'expliquer?

Une explication simple, et je dirai simpliste, est de penser qu'au dernier moment Pierre Vidal-Naquet n'a plus sous la main le récit de Josette Audin. En effet, le 9 avril Pierre Vidal-Naquet demande à Josette Audin de lui renvoyer son récit qui a été "stupidement égaré. " Mais la lecture des lettres de Josette Audin infirme cette explication : elle lui renvoie dès le 12 avril son récit. Toutefois Pierre Vidal-Naquet n'en accuse pas réception et n'y fait plus aucune allusion.

Une seconde explication s'accorde davantage à la méthode de l'historien. Depuis le début de cet échange de lettres Pierre Vidal-Naquet voit mal comment utiliser les documents partisans et les témoignages dans la rédaction de la brochure. Les documents partisans sont les appels, pétitions, tracts... émanant des camarades politiques de Josette Audin, les communistes d'Alger, et des syndicalistes. Étape par étape Pierre Vidal-Naquet va finalement totalement les éliminer de la brochure. On a là le premier exemple de ce qui sera une constante de son œuvre à venir : Pierre Vidal-Naquet ne récuse pas l'aspect militant que peut prendre le travail de l'historien, mais il condamne l'esprit partisan.

Les témoignages ont un statut différent de celui des documents partisans. Le témoignage du docteur Hadjadj, recueilli par Josette Audin, est reproduit dans le livre. Georges Hadjadj a vu Maurice Audin le 12 juin 1957 au Centre de triage d'El Biar où il était lui-même arrêté et où il avait été torturé. Puis le 18 juin, où Maurice Audin et Georges Hadjadj passent la journée dans la même pièce. Le 12 juin au soir Henri Alleg, directeur du quotidien Alger Républicain, voit également Maurice Audin.

41 Lettre J.A., 22 avril 1958.

42 Lettre P. V.N., 28 avril 1958. 
Il décrit ce moment dramatique dans son livre La question, passage qui est cité dans L'affaire Audin ${ }^{43}$.

Qu'en est-il du témoignage/récit de Josette Audin ? Il porte, selon les lettres, ces deux noms. Pierre Vidal-Naquet précise à plusieurs reprises ce qu'il attend de Josette Audin. Il s'agit de "présenter les faits dont vous avez été vous-même le témoin. Soit rédiger un récit minutieux des événements ${ }^{44}$ ». Mieux encore : "Il s'agit d'énumérer jour par jour et avec toutes les précisions imaginables les faits dont vous avez été le seul témoin ${ }^{45}$."

À ce moment de la rédaction de la brochure (décembre 1957 et début janvier 1958) le statut du " récit » de Josette Audin est important : "Un récit paraissant sous votre signature aurait plus de poids que tout autre ${ }^{46}$. " Et encore : "Votre récit est tout à fait ce qu'il faut avec quelques réserves. Permettez-moi d'en éliminer ce qui n'est pas témoignage mais appel à l'opinion publique ${ }^{47}$. " La possibilité d'insérer un appel, texte militant et politique, est encore évoquée plus tard par Josette Audin, mais cette insertion est refusée par le comité Audin ${ }^{48}$.

La première "censure " consiste à supprimer ce qui pourrait apparaître partisan dans le témoignage. Mais le 21 janvier le " récit " fait encore partie du plan de la brochure. Peu à peu chemine l'idée que l'historien peut ne pas se contenter de «faire la présentation et l'analyse critique des documents " travail dont devait se charger Pierre Vidal-Naquet ${ }^{49}$, mais qu'il doit écrire lui-même le récit des faits. Il est impossible de savoir exactement comment la décision de Pierre Vidal-Naquet a été prise, mais c'est un tournant important que le moment où l'historien prend en charge cette écriture. Le témoignage direct paraît finalement moins porteur de vérité que sa mise à distance par l'écriture de l'historien ${ }^{50}$. Le premier chapitre de L'affaire Audin : "La disparition de Maurice Audin ", rédigé par Pierre Vidal-Naquet, est en grande partie fondé sur le témoignage de Josette Audin, mais celui-ci n'est pas cité en tant que tel.

En contrepoint, Pierre Vidal-Naquet insiste tout au long de ses lettres sur les apports de son travail : " Mon travail apporte des certitudes. N'oubliez pas que la

43 H. Alleg, La Question, Paris, Minuit, 1958.

44 Lettre P. V.N., 26 décembre 1957.

45 Lettre P. V.N., 8 janvier 1958.

46 Lettre P. V.N., 26 décembre 1957.

47 Lettre P. V.N., 14 janvier 1958.

48 Lettre P. V.N., 28 avril 1958.

49 Lettre P. V.N., 26 décembre 1957.

50 Pierre Vidal-Naquet a souvent rencontré le problème du témoignage, de la mémoire et des mémoires dans son travail, en particulier dans ses écrits sur le monde juif. Il reconnaît qu'il y a tension entre vérité et mémoire, que la recherche de la vérité n'est pas la même chose que la transmission de la mémoire. Mais là encore l'historien par sa démarche enquête, analyse, réflexion - lui paraît particulièrement apte à savoir qu'il n'y a pas une mémoire pure, comme il n'y a pas une Vérité mais des vérités. L'effacement du témoignage de Josette Audin est le tout premier exemple de cette attitude critique. 
méthode historique existe ${ }^{51}$." "Mon travail ne fait appel à aucun document qui ne figure au dossier. C'est de l'interprétation de celui-ci par la méthode critique que naît la lumière. Je répète que celle-ci est aveuglante ${ }^{52}$."

Le récit de l'historien n'est pas celui du témoin. Et Pierre Vidal-Naquet reconnaît le caractère "froid " voire " glacé " de son texte. En écrivant cela il sous-entend sans doute qu'un récit de Josette Audin aurait conservé plus de chaleur. Mais cette perte de " chaleur " lui paraît constitutive de l'objectivité historique. De ceci bien sûr on pourrait discuter. Mais comme le dit dans une autre lettre Pierre Vidal-Naquet : "Ce livre ne tire sa force que de son objectivité scientifique ${ }^{53}$."

Tout ceci mérite bien sûr d'être nuancé, L'affaire Audin peut être lu aussi comme un livre engagé, L'affaire Audin aujourd'hui est un modèle de recherche de la vérité, L'affaire Audin est le fruit d'un dialogue entre deux personnes, respectueux, attentif et exemplaire. "Faire la lumière, établir la vérité ", tel est en effet le leitmotiv des lettres de Pierre Vidal-Naquet. La première lettre du 9 septembre 1957 donne le ton : « Je pense comme vous que les universitaires sauront exiger la lumière, non par simple esprit de confraternité mais par dignité d'hommes libres. »Et il conclut sa lettre ainsi : "Puisse la lumière être faite un jour pour l'honneur de notre pays. " Ce thème est repris plusieurs fois ${ }^{54}$. Dans une lettre du 8 janvier 1958 il écrit : "Je crois que nous arriverons à obtenir la vérité, non parce que le gouvernement dira ce qu'il sait, mais parce que notre pression à tous l'obligera à savoir et à dire. » Et un dimanche de mars 1958, à propos du texte d'un message qu'il a demandé à Josette Audin d'écrire pour qu'il soit lu lors d'un meeting de soutien : «Il n'est pas besoin d'un long texte. Il vous suffirait de rappeler que vous-même et toutes celles qui sont dans votre cas attendez de l'opinion démocratique française qu'elle sache exiger la vérité et la justice. » Et enfin dans la lettre du $1^{\text {er }}$ avril 1958 : "La vérité est en marche. "

Ce qui sera le fil directeur de toute l'œuvre et la vie de Pierre Vidal-Naquet : se mettre au service de la vérité, ce qu'il nomme lui-même plus tard " l'urgence de l'exigence de vérité », est bien présent dès ce moment. Mais cette recherche de la vérité ne peut se faire sans la confiance accordée à celui qui a pour tâche de révéler la vérité. C'est ce que réclame dans sa lettre du 24 novembre 1957 ce jeune historien de vingt-huit ans à Josette Audin :

51 Lettre P. V.N., 9 avril 1958.

52 Lettre P. V.N., 13 avril 1958.

53 Lettre P. V.N., 28 avril 1958.

54 Lettre P. V.N., 24 novembre 1957 : «Croyez que je n'épargnerai rien pour vous aider à faire la lumière dans cette affaire. " Lettre P. V.N., 26 décembre 1957 : "Croyez bien que de notre côté nous n'épargnerons rien pour connaître la vérité. " 
"Voulez-vous me faire confiance pour la rédaction de ce petit ouvrage ?... Je crois que cette confiance sera bien placée : je suis historien, fils de déportés morts à Auschwitz, et j'ai été élevé dans le souvenir de l'affaire Dreyfus ${ }^{55}$. "

Cette confiance, Josette Audin la lui accorde dans sa réponse du 26 novembre : " Je réponds de suite à votre lettre du 24 pour vous remercier d'accepter de vous occuper de la publication de la brochure dont vous me parlez. Je vous fais bien sûr confiance pour vous en charger ${ }^{56}$. " Et cette confiance ne se dément pas tout au long de cet échange de lettres, même si les deux correspondants ne sont pas toujours d'accord.

La lecture unilatérale des lettres de Pierre Vidal-Naquet pourrait laisser penser que l'accord entre les deux correspondants était total, à quelques nuances près. La lecture des lettres de Josette Audin laisse deviner des différences, surtout, me semblet-il, dans l'importance à donner au statut de militant communiste de Maurice Audin ${ }^{57}$ et au contexte général de résistance politique qui a pour but « que l'on mette fin à la guerre d'Algérie, et avec elle, à tous ses produits : les lieutenants Charbonnier et autres tortionnaires $^{58}$ ». Josette Audin recherche elle aussi la vérité, ce n'est pas un privilège réservé à l'historien ${ }^{59}$.

Dans l'échange des lettres entre Josette Audin et Pierre Vidal-Naquet, le sentiment de partager un même ensemble de valeurs se double peu à peu de chaleur et d'amitié. Cette amitié va s'exprimer dans le combat commun mené pendant des années pour faire éclater la vérité. Je cite la dernière lettre de cette correspondance, car elle dit mieux que tout la difficulté de la recherche de la vérité :

55 Lettre P. V.N., 24 novembre 1957.

56 Lettre J.A., 26 novembre 1957.

57 Dans sa lettre du 23 janvier 1958, Josette Audin écrit : « Je ne comprends pas bien votre ennui à écrire que Maurice Audin milite dans le PCA jusqu'à sa dissolution. Ceci n'exclut pas du tout qu'il ait continué à y militer après, mais alors c'était une action clandestine, donc inconnue en principe. Vous pouvez alors peut-être rajouter :

"Alors qu'il était étudiant il adhéra au Parti Communiste Algérien. Il y milita jusqu'à sa dissolution. Il était toujours d'accord avec les positions de ce parti, en particulier sur les 'événements d'Algérie' et la part que devaient y prendre les communistes."

Dites-moi ce que vous en pensez exactement. Il est certain que si on ne précise pas, de toute façon, tout le monde pensera que ce n'est pas un état de fait brutal, l'illégalité du PCA décidée du jour au lendemain, qui a pu modifier aussi brutalement l'attitude de quelqu'un qui a longuement réfléchi à ses pensées et ses actions. »

58 Lettre J.A., 22 avril 1958.

59 Lettre J.A., 2 janvier 1958 : «J'espère toujours que devant les protestations, on finira par dire cette vérité." 
" Jeudi 11 janvier 2006.

Chère Amie,

J'écris peu de lettres en ce début d'année, mais s'il y a quelqu'un à qui je me dois de répondre c'est bien vous. Dans deux ans notre amitié sera cinquantenaire et les assassins se taisent toujours.

Je vous embrasse vous et les vôtres,

Pierre Vidal-Naquet. "

Pauline Schmitt Pantel

Université Paris 1-Panthéon-Sorbonne

12, Place du Panthéon

F-75231 Paris Cedex 05

schmitt-pantel@wanadoo.fr 